\title{
On the Second Order Optimality Conditions for Optimization Problems with Inequality Constraints
}

\author{
Mourad Naffouti \\ Department of Mathematics, Faculty of Science, Mathematics, Physics and Natural of Tunis, Tunis, Tunisia \\ Email: mouradnaffouti@gmail.com
}

Received August 28, 2013; revised October 2, 2013; accepted October 27, 2013

Copyright (C) 2013 Mourad Naffouti. This is an open access article distributed under the Creative Commons Attribution License, which permits unrestricted use, distribution, and reproduction in any medium, provided the original work is properly cited.

\begin{abstract}
A nonlinear optimization problem $(P)$ with inequality constraints can be converted into a new optimization problem $(P E)$ with equality constraints only. This is a Valentine method for finite dimensional optimization. We review second order optimality conditions for $(P E)$ in connection with those of $(P)$. A strictly complementary slackness condition can be made to get the property that sufficient optimality conditions for $(P)$ imply the same property for $(P E)$. We give some new results (see Theorems 3.1, 3.2 and 3.3). Without any assumption, a counterexample is given to show that these conditions are not equivalent.
\end{abstract}

Keywords: Optimality Conditions; Constraint Qualifications; Copositivity

\section{Introduction}

Consider the following optimization problem

$$
(P)\left\{\begin{array}{l}
\min f(x) \\
\text { such that } \\
g_{i}(x) \leq 0, i \in I=\{1, \cdots, m\} \\
x \in \Omega
\end{array}\right.
$$

where $\Omega \subset \mathbb{R}^{n}$ is open and $f, g_{i} \Omega \rightarrow \mathbb{R}$ are $C^{2}$ functions.\#

Results on second order optimality conditions can be found in [1-3].\#

Converting inequality constraints into equality constraints, we get the following problem:

$$
(P E)\left\{\begin{array}{l}
\min f(x) \\
\text { such that } \\
g_{i}(x)+y_{i}^{2}=0, i \in I=\{1, \cdots, m\} \\
x \in \Omega, y \in \mathbb{R}^{m}
\end{array}\right.
$$

This method is known to be a Valentine method [4-6]. In the literature, second order optimality conditions for Valentine method are not studied.

Second order optimality conditions for $(P)$ are related to copositivity and are NP-hard [7]. Second order optimality conditions for $(P E)$ are related to the definiteness of a matrix in a vector subspace and there are efficient algorithms [8]. A strictly complementary slackness condition must be made to get the property that sufficient optimality conditions for $(P)$ imply the same property for $(P E)$.

Recall that the classical Karush-Kuhn-Tucker first and second order optimality conditions for a local minimizer $x^{*}$ for $(P)$, stated under the linear independence constraint qualification (LICQ), can be written as follows:

There exists a unique Lagrange multiplier $\lambda^{*}$ such that the Lagrangian function:

$$
L(x, \lambda)=f(x)+\sum_{i=1}^{m} \lambda_{i} g_{i}(x)
$$

Satisfies

$$
\left(C N_{1}\right)\left\{\begin{array}{l}
\nabla L\left(x^{*}, \lambda^{*}\right)=0 \\
g_{i}\left(x^{*}\right) \leq 0, \lambda_{i}^{*} \geq 0 \\
\lambda_{i}^{*} g_{i}\left(x^{*}\right)=0, \\
\forall i \in I=\{1, \cdots, m\}
\end{array}\right.
$$

And

$$
\left(C N_{2}\right) h^{T} \nabla_{x x}^{2} L\left(x^{*}, \lambda^{*}\right) h \geq 0, \forall h \in C\left(x^{*}\right)
$$

where $C\left(x^{*}\right)$ is the critical cone: if 


$$
I\left(x^{*}\right)=\left\{i / g_{i}\left(x^{*}\right)=0\right\}
$$

Then

$C\left(x^{*}\right)=\left\{h / f^{\prime}\left(x^{*}\right) h \leq 0, g_{i}^{\prime}\left(x^{*}\right) h \leq 0, \forall i \in I\left(x^{*}\right)\right\} \Leftrightarrow$ $C\left(x^{*}\right)=\left\{h / g_{i}^{\prime}\left(x^{*}\right) h \leq 0, \lambda_{i}^{*} g_{i}^{\prime}\left(x^{*}\right) h=0, \forall i \in I\left(x^{*}\right)\right\}$

The sufficient second order optimality condition for a feasible point $\bar{x}$ is: there exists a Lagrange multiplier $\bar{\lambda}$ such that

$$
\left(C S_{2}\right) h^{T} \nabla_{x x}^{2} L(\bar{x}, \bar{\lambda}) h>0, \forall h \in C\left(x^{*}\right), h \neq 0
$$

The fact that $\lambda^{*}$ or $\bar{\lambda}$ is the same for all critical vectors is very restrictive and, without (LICQ) or convexity assumptions, very difficult to get $[9,10]$. Recently, many authors have weakened the constraint qualification (LICQ) and $\left(\mathrm{CN}_{2}\right)$ are obtained [9-11]. In DaldoulBaccari [12], a numerical method is given in order to test the constant rank condition of Martinez et al. [11]. Another difficulty is that there is no efficient algorithm to test the conditions:

$$
\left(\mathrm{CN}_{2}\right) \text { or }\left(\mathrm{CS}_{2}\right) \text {. }
$$

Note that if $\lambda^{*}>0$ for all $i \in I\left(x^{*}\right)$ then $C\left(x^{*}\right)$ is a vector subspace and efficient algorithms for $\left(\mathrm{CN}_{2}\right)$ exist ([8]).

In this paper, we are interested by the use of efficient algorithms to test $\left(C N_{2}\right)$ or $\left(C S_{2}\right)$. A first step is the conversion of $(P)$ into $(P E)$.

Our main result is the following theorem. It is stated without any constraint qualification (linear independence, Mangasarian-Fromovitz or convexity assumptions).

Theorem 1.1. Let $x^{*}$ and $\left(x^{*}, y^{*}\right)$ be feasible points for

(P) and (PE) respectively where $y^{*}=\sqrt{-g_{i}\left(x^{*}\right)}$, then:

1) $x^{*}$ is a minimizer for $(P)$ if and only if $\left(x^{*}, y^{*}\right)$ is a minimizer for $(P E)$.

2) If a generalized Lagrange multiplier

$\left(\lambda_{0}, \lambda\right) \in \mathbb{R}_{+} \times \mathbb{R}_{+}^{m}$ satisfies the necessary second order optimality conditions for $(P)$ at $x^{*}$, then $\left(\lambda_{0}, \lambda\right)$ is a generalized Lagrange multiplier for $(P E)$ and satisfies the necessary second order optimality conditions at $\left(x^{*}, y^{*}\right)$

3) A generalized Lagrange multiplier

$\left(\lambda_{0}, \lambda\right) \in \mathbb{R}_{+} \times \mathbb{R}_{+}^{m}$ for (PE) satisfies sufficient second order optimality conditions at $\left(x^{*}, y^{*}\right)$ if and only if the following conditions hold:

a) $\left(\lambda_{0}, \lambda\right)$ is a generalized Lagrange multiplier for

b) $\lambda_{i}>0$ if $g_{i}\left(x^{*}\right)=0$

c) $\left(\lambda_{0}, \lambda\right)$ satisfies sufficient second order optimality conditions for $(P)$ at $x^{*}$

Note that
1) The minimizers $x^{*}$ and $\left(x^{*}, y^{*}\right)$ cited in the first item of Theorem 1.1 could be local or global.

2) The strict complementary slackness condition $\lambda_{i}>0$ if $g_{i}\left(x^{*}\right)=0$ is crucial in 3) and can be seen by the following simple counterexample:

$$
\left\{\min x^{2} / x \leq 0\right\}
$$

3) Existence of Lagrange multiplier in the second and third item of the theorem is not guaranteed [9].

We begin with some notations:

- The (generalized) Lagrangian function $\mathcal{L}$ of $(P)$ is defined on $\mathbb{R}^{n} \times \mathbb{R}_{+} \times \mathbb{R}_{+}^{m}$ by

$$
\mathcal{L}\left(x, \lambda_{0}, \lambda\right)=\lambda_{0} f(x)+\sum_{i=1}^{m} \lambda_{i} g_{i}(x)
$$

- The (generalized) Lagrangian function $\mathcal{L}_{0}\left(x, y, \lambda_{0}, \lambda\right)$ of $(P E)$ is defined on $\mathbb{R}^{n} \times \mathbb{R}^{m} \times \mathbb{R}_{+} \times \mathbb{R}_{+}^{m}$ by

$$
\mathcal{L}_{0}\left(x, y, \lambda_{0}, \lambda\right)=\lambda_{0} f(x)+\sum_{i=1}^{m} \lambda_{i}\left(g_{i}(x)+y_{i}^{2}\right)
$$

- The Lagrangian function $L$ of $(P)$ is

$$
L(x, \lambda)=\mathcal{L}(x, 1, \lambda)
$$

- The Lagrangian function $L_{0}$ of $(P E)$ is

$$
L_{0}(x, y, \lambda)=\mathcal{L}_{0}(x, y, 1, \lambda)
$$

- The gradient of $f$ with respect to $x$ is the column vector $\nabla f(x), \nabla f(x)^{T}$ is its transpose and the gradient of $\mathcal{L}$ with respect to $x$ is the column vector

$$
\nabla_{x} \mathcal{L}\left(x, \lambda_{0}, \lambda\right)=\lambda_{0} \nabla_{x} f(x)+\sum_{i=1}^{m} \lambda_{i} \nabla_{x} g_{i}(x)
$$

- The Hessian matrix of $\mathcal{L}$ with respect to $x$ is

$$
\nabla_{x x}^{2} \mathcal{L}\left(x, \lambda_{0}, \lambda\right)=\lambda_{0} \nabla_{x x}^{2} f(x)+\sum_{i=1}^{m} \lambda_{i} \nabla_{x x}^{2} g_{i}(x)
$$

- The set of feasible points of $(P)$ is

$$
\mathcal{F}=\left\{x \in \Omega / g_{i}(x) \leq 0, i \in I\right\}
$$

- The set of feasible points of $(P E)$ is

$$
\mathcal{F}_{0}=\left\{(x, y) \in \Omega \times \mathbb{R}^{m} / g_{i}(x)+y_{i}^{2}=0, i \in I\right\}
$$

- The critical cone at $x \in \mathcal{F}$ is

$$
C(P, x)=\left\{\begin{array}{l}
\mathrm{d} x \in \mathbb{R}^{n} / \nabla f(x)^{T} \mathrm{~d} x \leq 0, \nabla g_{i}(x)^{T} h \leq 0, \\
\forall i \in I(x)
\end{array}\right\}
$$

where

$$
I(x)=\left\{i \in / g_{i}(x)=0\right\}
$$

- The critical cone at $(x, y) \in \mathcal{F}_{0}$ is the subspace 


$$
C(P E, x, y)=\left\{\begin{array}{l}
\mathrm{d} X=(\mathrm{d} x, \mathrm{~d} y) / \nabla g_{i}(x)^{T} \mathrm{~d} x+2 y_{i} \mathrm{~d} y_{i}=0, \\
\forall i \in I(x)
\end{array}\right\}
$$

- For $x \in \mathcal{F}, \Lambda_{0}(P, x) \subset \mathbb{R}_{+} \times \mathbb{R}_{+}^{m}$ is the set of generalized Lagrange multipliers of $(P)$ at $x .\left(\lambda_{0}, \lambda\right) \in \Lambda_{0}(P, x)$ if and only if

$$
\left\{\begin{array}{l}
\left(\lambda_{0}, \lambda\right) \neq 0 \\
\nabla_{x} \mathcal{L}\left(x, \lambda_{0}, \lambda\right)=0 \\
\lambda_{i} \geq 0, \forall i \in I \\
\lambda_{i} g_{i}(x)=0, \forall i \in I
\end{array}\right.
$$

- The set of regular Lagrange multipliers of $(P)$ at a feasible point $x$ is

$$
\Lambda(P, x)=\left\{\lambda \in \mathbb{R}^{m} / \lambda_{0}=1,\left(\lambda_{0}, \lambda\right) \in \Lambda_{0}(P, x)\right\}
$$

- For $(x, y) \in \mathcal{F}_{0} \subset \mathbb{R}_{+} \times \mathbb{R}^{m}$, is the set of generalized Lagrange multipliers of $(P E)$ at $(x, y)$. $\left(\lambda_{0}, \lambda\right) \in \Lambda_{0}(P E, x, y)$ if and only if

$$
\left\{\begin{array}{l}
\left(\lambda_{0}, \lambda\right) \neq 0 \\
\nabla_{x} \mathcal{L}_{0}\left(x, y, \lambda_{0}, \lambda\right)=0
\end{array}\right.
$$

- The set of regular Lagrange multipliers of (PE) at $(x, y)$ is

$$
\Lambda(P E, x, y)=\left\{\lambda \in \mathbb{R}^{m} / \lambda_{0}=1,\left(\lambda_{0}, \lambda\right) \in \Lambda_{0}(P E, x, y)\right\}
$$

- The set of normalized Lagrange multipliers of $(P)$ at $x^{*} \in \mathcal{F}_{0}$ is

$$
\Lambda_{1}\left(P, x^{*}\right)=\left\{\left(\lambda_{0}, \lambda\right) \in \Lambda_{0}\left(P, x^{*}\right) / \lambda_{0}+\sum_{i=1}^{m} \lambda_{i}=1\right\}
$$

- The set of normalized Lagrange multipliers of (PE) at $X^{*}=\left(x^{*}, y^{*}\right) \in \mathcal{F}_{0}$ is

$$
\Lambda_{1}\left(P E, x^{*}, y^{*}\right)=\left\{\left(\lambda_{0}, \lambda\right) \in \Lambda_{0}\left(P E, x^{*}, y^{*}\right) / \lambda_{0}+\sum_{i=1}^{m}\left|\lambda_{i}\right|=1\right\}
$$

- $x \in \mathcal{F}$ satisfies the strictly complementary slackness condition if the following condition holds.

$$
(S C S) \exists\left(\lambda_{0}, \lambda\right) \in \Lambda_{0}(P, x), \lambda_{i}>0, \forall i / g_{i}(x)=0
$$

- Necessary first and second order optimality conditions for $(P)$ can be written in the following form ([13], Theorem 9.3).

Theorem 1.2. If $x^{*}$ is a local minimizer for $(P)$ then:

$$
\begin{gathered}
\Lambda_{0}(P, x) \neq \varnothing \\
\forall \mathrm{d} x \in C(P, x), \exists\left(\lambda_{0}, \lambda\right) \in \Lambda_{0}\left(P, x^{*}\right) \\
/ \mathrm{d} x^{T} \nabla_{x x}^{2} \mathcal{L}\left(x^{*}, \lambda_{0}, \lambda\right) \mathrm{d} x \geq 0 .
\end{gathered}
$$

The necessary second order optimality conditions (1.2) can be written as

$$
\begin{aligned}
& \max _{\left(\lambda_{0}, \lambda\right) \in \Lambda_{1}\left(P, x^{*}\right)} \mathrm{d} x^{T} \nabla_{x x}^{2} \mathcal{L}\left(x^{*}, \lambda_{0}, \lambda\right) \mathrm{d} x \geq 0 . \\
& \forall \mathrm{d} x \in C\left(P, x^{*}\right) .
\end{aligned}
$$

In the same way, for a local minimizer $X^{*}=\left(x^{*}, y^{*}\right)$ of $(P E)$, we have

$$
\begin{gathered}
\Lambda_{0}\left(P E, X^{*}\right) \neq \varnothing \\
\max _{\left(\lambda_{0}, \lambda\right) \in \Lambda_{1}\left(P E, X^{*}\right)} \mathrm{d} X^{T} \nabla_{X X}^{2} \mathcal{L}\left(X^{*}, \lambda_{0}, \lambda\right) \mathrm{d} X \geq 0 . \\
\forall \mathrm{d} X \in C\left(P E, X^{*}\right) .
\end{gathered}
$$

- For $x^{*} \in \mathcal{F}$ the generalized sufficient second order optimality conditions, $\left(\operatorname{GSC}_{2}\left(P, x^{*}\right)\right)$, for $(P)$ are that $\Lambda_{0}\left(P, x^{*}\right)$.

Or $\Lambda_{1}\left(P, x^{*}\right)$ are not empty and, for every $\mathrm{d} x \in C\left(P, x^{*}\right), \mathrm{d} x \neq 0$, one has

$$
\max _{\left(\lambda_{0}, \lambda\right) \in \Lambda_{1}\left(P, x^{*}\right)} \mathrm{d} x^{T} \nabla_{x x}^{2} \mathcal{L}\left(x^{*}, \lambda_{0}, \lambda\right) \mathrm{d} x>0 .
$$

For $X^{*} \in \mathcal{F}_{0}$, the generalized sufficient second order optimality conditions, $\left(G S C_{2}\left(P E, X^{*}\right)\right)$ for $(P E)$ are that $\Lambda_{0}\left(P E, X^{*}\right)$ or $\Lambda_{1}\left(P E, X^{*}\right)$ are not empty and, for every $\mathrm{d} X \in C\left(P E, X^{*}\right), \mathrm{d} X \neq 0$, one has

$$
\max _{\left(\lambda_{0}, \lambda\right) \in \Lambda_{1}\left(P E, X^{*}\right)} \mathrm{d} X^{T} \nabla_{x x}^{2} \mathcal{L}\left(X^{*}, \lambda_{0}, \lambda\right) \mathrm{d} X>0 .
$$

- The classical sufficient second order optimality condition for $(P)$, at $x^{*} \in \mathcal{F}$, is that there exists $\lambda^{*} \in \Lambda\left(P, x^{*}\right)$ such that

$$
\mathrm{d} x^{T} \nabla_{x x}^{2} L\left(x^{*}, \lambda^{*}\right) \mathrm{d} x>0, \forall \mathrm{d} x \in C\left(P, x^{*}\right), \mathrm{d} x \neq 0
$$

- In the same way, the classical sufficient second order optimality condition for $(P E)$, at $X^{*} \in \mathcal{F}_{0}$, is that there exists $\lambda^{*} \in \Lambda\left(P E, X^{*}\right)$ such that $\mathrm{d} X^{T} \nabla_{x x}^{2} L\left(X^{*}, \lambda^{*}\right) \mathrm{d} X>0, \forall \mathrm{d} x \in C\left(P E, X^{*}\right), \mathrm{d} X \neq 0$

In $[14,15]$, the existence of such multipliers is studied.

- We say that $\left(\lambda_{0}, \lambda\right) \in \Lambda\left(P, x^{*}\right)$ satisfies the sufficient second order optimality conditions for $(P)$ if

$$
\mathrm{d} x^{T} \nabla_{x x}^{2} \mathcal{L}\left(x^{*}, \lambda_{0}, \lambda\right) \mathrm{d} x>0, \forall \mathrm{d} x \in C\left(P, x^{*}\right), \mathrm{d} x \neq 0
$$

In the same way, $\left(\lambda_{0}, \lambda\right) \in \Lambda\left(P E, X^{*}\right)$ satisfies the sufficient second order optimality conditions for $(P E)$ if

$$
\mathrm{d} X^{T} \nabla_{x x}^{2} \mathcal{L}_{0}\left(X^{*}, \lambda_{0}, \lambda\right) \mathrm{d} X>0, \forall \mathrm{d} X \in C\left(P E, X^{*}\right), \mathrm{d} X \neq 0
$$

\section{Some Properties of (PE) and (P)}

Let $x^{*}$ be a local minimizer for $(P)$ and $X^{*}$ the corresponding minimizer for $(P E)$. It is easy to see that 


$$
\Lambda_{0}\left(P, x^{*}\right) \subset \Lambda_{0}\left(P E, X^{*}\right)
$$

The following properties are easy to check:

- If $\mathrm{d} X=(\mathrm{d} x, \mathrm{~d} y) \in C\left(P E, X^{*}\right)$ and

$C_{0}\left(P, x^{*}\right)=\left\{d / \nabla f\left(x^{*}\right)^{T} d \leq 0, \nabla g\left(x^{*}\right)^{T} d=0, i \in I\left(x^{*}\right)\right\}$

Then

$$
\mathrm{d} x \in C_{0}\left(P, x^{*}\right) \subset C\left(P, x^{*}\right)
$$

And

$$
(W S C S) \Rightarrow C_{0}\left(P, x^{*}\right) \subset C\left(P, x^{*}\right)
$$

- If $\mathrm{d} x \in C_{0}\left(P, X^{*}\right)$ then there exists $d y$ such that

$$
\mathrm{d} X=(\mathrm{d} x, \mathrm{~d} y) \in C\left(P E, X^{*}\right)
$$

- If $x \in \mathcal{F}$ satisfies the linear independence constraint qualification (LICQ), so is $X=(x, y) \in \mathcal{F}_{0}$

- It is easy to see that

$$
\left(\lambda_{0}, \lambda\right) \in \Lambda_{0}\left(P E, X^{*}\right) \Rightarrow \lambda_{i}=0, \forall i \notin I\left(x^{*}\right)
$$

\section{Optimality under Regularity}

We begin with the regular case and extend the result of ([16], Proposition 1.32).

Theorem.3.1. Let $x^{*}$ be a feasible point for $(P)$, satisfies (LICQ) and (SCS), then the classical sufficient second order optimality condition (1.8) holds if and only if the classical sufficient second order optimality condition (1.9) holds.

Proof. $x^{*}$ satisfies (LICQ) and $\Lambda\left(P, x^{*}\right)$ is a singleton $\left(\Lambda\left(P, x^{*}\right)=\{\lambda\}\right)$.

Also $\Lambda\left(P E, X^{*}\right)=\{\lambda\}$ and, from (SCS), we have

$$
\lambda_{i}^{*}>0, \forall i \in I\left(x^{*}\right)
$$

The first part of the theorem is the Proposition 1.32 of [16]. To prove the "only if", Let $\mathrm{d} x \in C\left(P, x^{*}\right)$ and $\mathrm{d} x \neq 0$. Put $\mathrm{d} y_{i}=0$ if $i \in I\left(x^{*}\right)$ and

$$
\mathrm{d} y_{i}=-\frac{\nabla g_{i}\left(x^{*}\right)^{T} \mathrm{~d} x}{2 y_{i}^{*}}
$$

If $i \notin I\left(x^{*}\right)$. We have $\mathrm{d} X=(\mathrm{d} x, \mathrm{~d} y) \in C\left(P E, X^{*}\right)$ and $\mathrm{d} X \neq 0$. From $\lambda_{i}=0, \forall i \notin I\left(x^{*}\right)$, we get

$$
0<\mathrm{d} X^{T} \nabla_{x x}^{2} L_{0}\left(X^{*}, \lambda^{*}\right) \mathrm{d} X=\mathrm{d} x^{T} \nabla_{x x}^{2} L\left(x^{*}, \lambda^{*}\right) \mathrm{d} x .
$$

In the above theorem, (LICQ) is not necessary and one can prove the following theorem (see [16], Proposition 1.31).

Theorem.3.2. Let $\left(\lambda_{0}^{*}, \lambda^{*}\right) \in \Lambda_{0}\left(P, X^{*}\right)$ such that

$$
\lambda_{i}^{*}>0, \forall i \in I\left(x^{*}\right)
$$

Then $\left(\lambda_{0}^{*}, \lambda^{*}\right)$ satisfies the sufficient second order optimality conditions for $(P)$ if and only if it satisfies the sufficient second order optimality conditions for (PE) hold.

The main result of this section is the following theorem (compare with [16], Proposition 1.32).

Theorem.3.3. Let $x^{*}$ be a feasible point for $(P)$ and such that that

1) There exists a multiplier $\left(\lambda_{0}^{*}, \lambda^{*}\right) \in \Lambda_{0}\left(P, X^{*}\right)$ such

$$
\lambda_{i}^{*}>0, \forall i \in I\left(x^{*}\right)
$$

2) The sufficient second order optimality condition $\left(\operatorname{GSC}_{2}\left(P, x^{*}\right)\right)$ hold

Then $X^{*}=\left(x^{*}, y^{*}\right)$ satisfies the sufficient second order optimality conditions $\left(G S C_{2}\left(P E, X^{*}\right)\right)$.

Proof. Let $\mathrm{d} X=(\mathrm{d} x, \mathrm{~d} y) \in C\left(P E, X^{*}\right), \mathrm{d} X \neq 0$. We know that $\mathrm{d} x \in C\left(P, x^{*}\right)$ and we have two cases:

1) $\mathrm{d} x=0$. This means that $\mathrm{d} y \neq 0, \mathrm{~d} y_{i}=0$ for all $i \notin I\left(x^{*}\right)$ and

$$
\mathrm{d} X^{T} \nabla_{x x}^{2} \mathcal{L}_{0}\left(X^{*}, \lambda_{0}^{*}, \lambda^{*}\right) \mathrm{d} X=2 \sum_{i \in I\left(x^{*}\right)} \lambda_{i}^{*}\left(\mathrm{~d} y_{i}\right)^{2}>0
$$

2) $d x \neq 0$. From $\left(\operatorname{GSC}_{2}\left(P, x^{*}\right)\right)$, there exists $\left(\lambda_{0}, \lambda\right) \in \Lambda_{1}\left(P, x^{*}\right)$ such that

$$
\mathrm{d} x^{T} \nabla_{x x}^{2} \mathcal{L}\left(x^{*}, \lambda_{0}, \lambda\right) \mathrm{d} x>0
$$

And

$$
\begin{aligned}
& \mathrm{d} X^{T} \nabla_{x x}^{2} \mathcal{L}_{0}\left(X^{*}, \lambda_{0}, \lambda\right) \mathrm{d} X \\
& =\mathrm{d} x^{T} \nabla_{x x}^{2} \mathcal{L}\left(x^{*}, \lambda_{0}, \lambda\right) \mathrm{d} x+2 \sum_{i \in I\left(x^{*}\right)} \lambda_{i}^{*}\left(\mathrm{~d} y_{i}\right)^{2} \\
& >0
\end{aligned}
$$

\section{A Counterexample}

The following counterexample shows that $\left(G_{2} C_{2}\left(P E, X^{*}\right)\right)$, do not imply

$$
\left(\operatorname{GSC}_{2}\left(P, x^{*}\right)\right) \text {, }
$$

\section{Example 4.1.}

$$
(P)\left\{\begin{array}{l}
\min z^{2} \\
\text { such that } \\
g_{i}(x, y)-i z \leq 0, \quad i \in I=\{1, \cdots, 6\} \\
x, y, z \in \mathbb{R}
\end{array}\right.
$$

where

$$
\begin{gathered}
g_{1}(x, y)=2 \sqrt{3} x y-2 y^{2} \\
g_{2}(x, y)=-2 \sqrt{3} x y-2 y^{2} \\
g_{3}(x, y)=y^{2}-3 x^{2}
\end{gathered}
$$




$$
\begin{gathered}
g_{4}(x, y)=2 \sqrt{3} x y-2 x^{2} \\
g_{5}(x, y)=-2 \sqrt{3} x y-2 x^{2} \\
g_{6}(x, y)=x^{2}-3 y^{2}
\end{gathered}
$$

We list some properties of $(P)$ :

1) $g_{1}(x, y) \cdot g_{2}(x, y)=4 y^{2} g_{3}(x, y)$ and

$$
g_{4}(x, y) \cdot g_{5}(x, y)=4 x^{2} g_{6}(x, y)
$$

2) $z \geq 0 \Rightarrow(0,0, z) \in \mathcal{F}, x^{*}=(0,0,0)$ is a minimizer for $(P)$

3) $C\left(P, x^{*}\right)=\left\{d=(x, y, z) \in \mathbb{R}^{3} / z \leq 0\right\} \neq(0,0,0)$

4) $\Lambda\left(P, x^{*}\right)=\{(0,0,0)\}$ and $\left(G_{2} C_{2}\left(P, x^{*}\right)\right)$, do not hold.

Consider the associated $(P E)$ problem:

$$
(P E)\left\{\begin{array}{l}
\min z^{2} \\
\text { such that } \\
g_{i}(x, y)-i z u_{i}^{2}=0, i \in I=\{1, \cdots, 6\} \\
x, y, z, u_{i} \in \mathbb{R}
\end{array}\right.
$$

Its generalized Lagrangian function is, with $X=(x, y, z, u)$,

$$
\mathcal{L}_{0}\left(X, \lambda_{0}, \lambda\right)=\lambda_{0} z^{2}+\sum_{i=1}^{6} \lambda_{i}\left(g_{i}(x, y)-i z+u_{i}^{2}\right) .
$$

$X^{*}=\left(x^{*}, 0\right)$ is a minimizer for $(P E)$ and we get

$$
\begin{gathered}
\nabla \mathcal{L}_{0}\left(X^{*}, \lambda_{0}, \lambda\right)=0 \Rightarrow \sum_{i=1}^{6} i \lambda_{i}=0 . \\
\Lambda_{0}\left(P E, X^{*}\right)=\left\{\left(\lambda_{0}, \lambda\right) \neq 0 / \lambda_{0} \geq 0, \sum_{i=1}^{6} i \lambda_{i}=0\right\} \\
C\left(P E, X^{*}\right)=\left\{d=(x, y, z, u) \in \mathbb{R}^{9} / z=0\right\} \\
d \in C\left(P E, X^{*}\right) \Rightarrow \\
Q(d)=d^{T} \nabla_{x x}^{2} \mathcal{L}_{0}\left(X^{*}, \lambda_{0}, \lambda\right) d \\
=2 \lambda_{1}\left(2 \sqrt{3} x y-2 y^{2}\right)+2 \lambda_{2}\left(-2 \sqrt{3} x y-2 y^{2}\right) \\
+2 \lambda_{3}\left(y^{2}-3 x^{2}\right)+2 \lambda_{4}\left(2 \sqrt{3} x y-2 x^{2}\right) \\
+2 \lambda_{5}\left(-2 \sqrt{3} x y-2 x^{2}\right)+2 \lambda_{6}\left(-2 \sqrt{3} x y-2 y^{2}\right)
\end{gathered}
$$

We can prove

Lemma 4.2.

For every $d \in C\left(P E, X^{*}\right), d=(x, y, z, u) \neq 0$, there exists a multiplier $\left(\lambda_{0}, \lambda\right) \in \Lambda_{0}\left(P E, X^{*}\right)$ such that

$$
Q(d)=d^{T} \nabla_{x x}^{2} \mathcal{L}_{0}\left(X^{*}, \lambda_{0}, \lambda\right) d>0
$$

Proof. It is easy to see that

$$
\begin{aligned}
& d \in C\left(P E, X^{*}\right) \\
& \Rightarrow d^{T} \nabla_{x x}^{2} \mathcal{L}_{0}\left(X^{*}, \lambda_{0}, \lambda\right)=2 \sum_{i=1}^{6} \lambda_{i}\left(g_{i}(x, y)+u_{i}^{2}\right) .
\end{aligned}
$$

We prove, first, that

$$
g_{1}(x, y)+u_{i}^{2} \leq 0, \forall i \Rightarrow d=0 .
$$

This is true if $x=0$ or $y=0$. Suppose that $x \neq 0$ and $y \neq 0$ and

$$
g_{1}(x, y)+u_{i}^{2} \leq 0, \forall i=1, \cdots, 6
$$

We get

$$
g_{1}(x, y) \leq 0, \forall i=1, \cdots, 6
$$

and, from 1), we obtain

$$
x=y=0, u_{i}=0, \forall i=1, \cdots, 6
$$

and this contradicts $x \neq 0$ and $y \neq 0$. So

$$
x=y=0, u_{i}=0, \forall i=1, \cdots, 6
$$

We conclude that we have two cases:

1) $a_{i}=g_{i}(x, y)+u_{i}^{2}>0, \forall i=1, \cdots, 6$ we have two cases

i) There exists $i<j$ such that $a_{i}=a_{j}=a$. put $\lambda_{i}=j, \lambda_{j}=-i, \lambda_{k}=0, k \notin\{i, j\}, \lambda_{0}=1$ we get

$$
i \lambda_{i}+j \lambda_{j}=0
$$

This means that

$$
\left(\lambda_{0}, \lambda\right) \in \Lambda_{0}\left(P E, X^{*}\right)
$$

And

$$
Q(d)=2\left(\lambda_{i} a_{i}+\lambda_{j} a_{j}\right)=2 a(j-i)>0
$$

ii) $a_{i} \neq a_{j}, \forall j \neq i$. We have two cases

a) There exists $i<j$ such that $-j \lambda_{i}+i \lambda_{j} \neq 0$ We can choose $\lambda_{j}$ so that $i \lambda_{i}+j \lambda_{j}=0$ and for $\lambda_{0}=1, \lambda_{k}=0, k \notin\{i, j\}$, we get $\left(\lambda_{0}, \lambda\right) \in \Lambda_{0}\left(P E, X^{*}\right)$ and

$$
\begin{aligned}
& Q(d)=2\left(\lambda_{i} a_{i}+\lambda_{j} a_{j}\right)=2 \lambda_{j}\left(-\frac{j}{i} a_{i}+a_{j}\right) \\
& =2 \frac{\lambda_{j}}{i}\left(-j a_{i}+i a_{j}\right)>0
\end{aligned}
$$

b) $-j a_{i}+i a_{j}=0, i \neq j$, we get $a_{i}=i a_{1}, a_{1}<a_{2}<a_{3}$ and $\lambda_{1}=\lambda_{2}=-1 ; \lambda_{3}=1$ satisfy $\lambda_{1}+2 \lambda_{2}+3 \lambda_{3}=0$.

For $\lambda_{0}=1, \lambda_{k}=0, k \notin\{i, j\}$, we get $\left(\lambda_{0}, \lambda\right) \in \Lambda_{0}\left(P E, X^{*}\right)$ and satisfy

$Q(d)=2\left(\lambda_{1} a_{1}+\lambda_{2} a_{2}+\lambda_{3} a_{3}\right)=2\left(-a_{1}+2 a_{2}+3 a_{3}\right)>0$

2) There exists $i$ such that a $a_{i}<0$ and $j$ such that $a_{j}>0$, it is easy to find $\lambda_{i}<0$ and $\lambda_{j}>0$, such that 


$$
i \lambda_{i}+j \lambda_{j}=0
$$

For $\lambda_{0}=1, \lambda_{k}=0, k \notin\{i, j\}$, we get

$$
\left(\lambda_{0}, \lambda\right) \in \Lambda_{0}\left(P E, X^{*}\right)
$$

And satisfies

$$
Q(d)=2\left(\lambda_{i} a_{i}+\lambda_{j} a_{j}\right)>0 .
$$

\section{Proof of Theorem 1.1.}

1) Suppose $x^{*}$ is a local minimizer for $(P)$. There exists an open ball $B\left(x^{*}, r\right) \subset \Omega$ such that

$$
\left\{x \in B\left(x^{*}, r\right), g_{i}(x) \leq 0, i \in I\right\} \Rightarrow f\left(x^{*}\right) \leq f(x)
$$

Let $\left(x^{*}, y^{*}\right)$ be the corresponding feasible point for $(P E)$, that is $y_{i}=\sqrt{-g_{i}\left(x^{*}\right)} \cdot V=B\left(x^{*}, r\right) \cap \mathbb{R}^{m}$ is open and $\left(x^{*}, y^{*}\right) \in V$. We get

$$
\begin{aligned}
& \left\{(x, y) \in V, g_{i}(x)+y_{i}^{2}=0, i \in I\right\} \\
& \Rightarrow\left\{x \in B\left(x^{*}, r\right), g_{i}(x) \leq 0, i \in I\right\} \\
& \Rightarrow f\left(x^{*}\right) \leq f(x) .
\end{aligned}
$$

Now, suppose that $\left(x^{*}, y^{*}\right)$ is local minimizer for $(P E)$, there exists $r_{1}>0, r_{2}>0$ such that

$$
\begin{aligned}
& \left\{(x, y) \in B\left(x^{*}, r_{1}\right) \times B\left(y^{*}, r_{2}\right), g_{i}(x)+y_{i}^{2}=0, i \in I\right\} \\
& \Rightarrow f\left(x^{*}\right) \leq f(x)
\end{aligned}
$$

2) We know that $\lambda_{i}=0$ if $g_{i}\left(x^{*}\right)<0$. Suppose that $i=1 \in I\left(x^{*}\right)$ and let $\mathrm{d} y_{i}=0, i \in I, i \neq 1$ and $\mathrm{d} x=0$. For all $\mathrm{d} y_{1} \in \mathbb{R}, \mathrm{d} X=(\mathrm{d} x, \mathrm{~d} y) \in C\left(P E,\left(x^{*}, y^{*}\right)\right)$ and $0 \leq \mathrm{d} X^{T} \nabla_{x x}^{2} \mathcal{L}_{0}\left(X^{*}, \lambda_{0}, \lambda\right) \mathrm{d} X=2 \lambda_{1}\left(\mathrm{~d} y_{1}\right)^{2}$. We get that $\lambda_{1}>0$ and this is true for any $\lambda_{i}$

3) Suppose that $\left(\lambda_{0}, \lambda\right)$ satisfies the sufficient second order optimality conditions for $(P E)$. This means that for all $0 \neq \mathrm{d} X=(\mathrm{d} x, \mathrm{~d} y) \in C\left(P E,\left(x^{*}, y^{*}\right)\right)$, we have

$$
\begin{aligned}
& \mathrm{d} X^{T} \nabla_{x x}^{2} \mathcal{L}_{0}\left(X^{*}, \lambda_{0}, \lambda\right) \mathrm{d} X \\
& =\mathrm{d} x^{T} \nabla_{x x}^{2} \mathcal{L}\left(x^{*}, \lambda_{0}, \lambda\right) \mathrm{d} x+2 \sum_{i \in I\left(x^{*}\right)} \lambda_{i}\left(\mathrm{~d} y_{i}\right)^{2} \\
& >0
\end{aligned}
$$

Suppose that $i=1 \in I\left(x^{*}\right)$ and let $\mathrm{d} y_{i}=0, i \in I, i \neq 1$ and $\mathrm{d} x=0$. For all $\mathrm{d} y_{1} \in \mathbb{R}, \mathrm{d} X=(\mathrm{d} x, \mathrm{~d} y) \in C\left(P E,\left(x^{*}, y^{*}\right)\right)$ and

$$
0<\mathrm{d} X^{T} \nabla_{x x}^{2} \mathcal{L}_{0}\left(X^{*}, \lambda_{0}, \lambda\right) \mathrm{d} X=2 \lambda_{1}\left(\mathrm{~d} y_{1}\right)^{2} .
$$

We get that $\lambda_{1}>0$ and this is true for any $\lambda_{i}, i \in I\left(x^{*}\right)$.

\section{Concluding Remarks}

In the regular case and in the presence of strictly complementary slackness (SCS), we have shown that an optimization problem $(P)$, with inequality constraints, can be converted into an optimization problem $(P E)$ with equality constraints in such a way that sufficient second order optimality conditions are preserved. Without any regularity assumption, we have shown that sufficient second order optimality conditions hold for $(P E)$ if these hold for $(P)$ and if (SCS) holds.

\section{REFERENCES}

[1] F. Jhon, "Extremum Problems with Inequalities as Side Conditions, Studies and Essays, Courant Anniversary Volume,” Wiley-Interscience, Hoboken, 1948.

[2] E. S. Levitin, A. A. Milyutin and N. P. Osmolovskii, "Conditions of High Order for a Local Minimum in Problems with Constraints,” Russian Mathematical Surveys, Vol. 33, No. 6, 1978, pp. 97-168.

[3] A. Ioffe, "Necessary and Sufficient Conditions for a Local Minimum. 3: Second Order Conditions and Augmented Duality," SIAM Journal of Control and Optimization, Vol. 17, No. 2, 1979, pp. 266-288.

[4] F. A. Valentine, "The Problem of Lagrange with Differentiable Inequalities as Side Consrtaints, Contribution to the Calculus of Variation 1933-1937," University of Chicago Press, Chicago, 1937, pp. 407-448.

[5] J. B. Hiriart-Urruty, "Optimisation et Analyse Convexe, Exercices Corrigées,” EDP Sciences, 2009.

[6] L. D. Berkovitz, "Variational Methods of Control and Programming," Journal of Mathematical Analysis and Applications, Vol. 3, No. 1, 1961, pp. 145-169. http://dx.doi.org/10.1016/0022-247X(61)90013-0

[7] K. G. Murty and S. N. Kabadi, "Some NP-Complete Problems in Quadratic and Nonlinear Programming," Mathematical Programming, Vol. 39, No. 2, 1987, pp. 117-129. http://dx.doi.org/10.1007/BF02592948

[8] Y. Chabrillac and J. P. Crouzeix, "Definiteness and Semidefiniteness of Quadratic Forms Revisited,” Linear Algebra and Its Applications, Vol. 63, No. 1, 1984, pp. 283292. http://dx.doi.org/10.1016/0024-3795(84)90150-2

[9] A. Baccari, "On the Classical Necessary Second-Order Optimality Conditions,” Journal of Optimization Theory and Applications, Vol. 123, No. 1, 2004, pp. 213-221. http://dx.doi.org/10.1023/B:JOTA.0000043998.04008.e6

[10] A. Baccari and A. Trad, “On the Classical Necessary Second-Order Optimality Conditions in The Presence of Equality and Inequality Constraints," SIAM. Journal of Optimization, Vol. 15, No. 2, 2004, pp. 394-408. http://dx.doi.org/10.1137/S105262340342122X

[11] R. Andreani, J. M. Martinez and M. L. Schuverdt, “On Second-Order Optimality Conditions for Nonliear Programming," Optimization, Vol. 56, No. 5-6, 2007, pp. 529-542.

[12] M. Daldoul and A. Baccari, “An Application of Matrix Computations to Classical Second-Order Optimality Con- 
ditions," Optimization Letters, Vol. 3, No. 4, 2009, pp. 547-557. http://dx.doi.org/10.1007/s11590-009-0134-9

[13] A. Ben-Tal and J. Zowe, "A Unified Theory of First and Second Order Conditions for Extremum Problems in Topological Vector Spaces,” Mathematical Programming Study, Vol. 19, 1982, pp. 39-76.

http://dx.doi.org/10.1007/BFb0120982

[14] J. F. Bonnans and A. Shapiro, "Perturbation Analysis of
Optimization Problems,” Springer, Berlin, 2000.

[15] O. L. Mangasarian and S. Fromovitz, "The Fritz John Necessary Optimality Conditions in the Presence of Equality and Inequality Constraints,” Journal of Mathematical Analysis and Applications, Vol. 17, 1967, pp. 3747.

[16] D. P. Bertsekas, "Constrained Optimization and Lagrange Multiplier Methods,” Academic Press, Cambridge, 1982. 